\title{
Implant retained or conventional dentures, which give more patients satisfaction?
}

\author{
Are implant-retained mandibular overdentures better than conventional \\ complete dentures?
}

\begin{abstract}
Allen PF, Thomason JM, Jepson NJ, NohI F, Smith DG, Ellis J. A randomized controlled trial of implant-retained mandibular overdentures. J Dent Res 2006; 85:547-551
\end{abstract}

Design This was a randomised controlled trial (RCT) set in a dental hospital.

Intervention The implant group (IG) had two implants placed in the interforaminal region of the lower jaw followed (after healing) by a denture fixed to the implants by a ball attachment mechanism. In the conventional dentures group (CG), dentures were constructed using conventional replacement denture techniques. Patients in the IG had conventional upper dentures made in the same fashion.

Outcome measure The performance of the dentures was evaluated using an oral health impact profile (OHIP) and a denture satisfaction scale before treatment and 3 months post-treatment.

Results Analysis was conducted on an intention-to-treat basis. Substantial improvements in oral-health-related quality of life and denture satisfaction were reported by both groups. There were, however, no significant differences post-treatment between the groups. Patients randomised to the IG who declined implants had significantly lower pretreatment OHIP scores and there were significantly greater pre-/ post-treatment change-scores for individuals who had implants compared with the change-scores of people who declined.

Conclusions There were no significant post-treatment differences between the groups, but a treatment effect may be masked by the intention-to-treat analysis. The pre-/ post-treatment OHIP change-scores were significantly greater for people receiving implants than for those who refused.
Address for correspondence: Dr PF Allen, University Dental School and Hospital, Wilton, Cork, Ireland. E-mail: f.allen@ucc.ie

\section{Commentary}

This very interesting study is the first RCT in dentistry where the post-randomised single-consent design described by Zelen in $1979^{1}$ has been used. The rationale for introducing a study design where the participants are randomised before acquiring consent was that it might enhance patient recruitment to clinical trials. Interestingly, not many trials in biomedicine have applied this design, and the majority of those that have suggest that the reason is to avoid bias due to a Hawthorne effect a distortion of the results caused by the response the participants to the special attention they receive from researchers rather than to improve recruitment. ${ }^{2}$

Many, if not most, interventions in restorative dentistry and prosthodontics can be considered as elective. For many potential patients, the most relevant treatment outcomes are previous patients' satisfaction with the treatment and how it may affect their quality of life. In this context it is important to present to the patient unbiased evidence from appropriately designed clinical studies, to enable him or her to consent to a particular treatment strategy. Unfortunately, however, there is a paucity of research data available. It is also questionable whether such data, arising from participants in stringently controlled clinical studies, are applicable to all. The reason for this is that voluntary participation in clinical trials is influenced by patients' prior expectations and general attitudes towards dental care and research. ${ }^{3}$ Thus, people who volunteer to participate, for example, in RCT, which are inherently associated with an element of risk, may not be representative of others who may have strong treatment preferences, for right or wrong reasons of their own. It is unfortunate therefore that there are so few trials in the dental literature that have used those alternative designs that take into account participants' preferences. Notable exceptions are the trials and publications by Feine and colleagues in Montreal, Canada. ${ }^{4}$

One of the intriguing findings in this trial is the relatively high proportion of individuals (12 out of $45 ; 25 \%$ ) who did not accept receiving an "expensive, high quality intervention", ie, the implant-supported overdenture instead of simple denture. Risking a high proportion of refusals is in fact one of the main criticisms of the Zelen design, because statistical power is lost. This adds to the already controversial ethical dilemmas associated with this study design. $^{2}$

Could this high dropout have been predicted? Perhaps not, as a similar occurrence also puzzled the authors of another recent implant trial. ${ }^{5}$ In the latter, approximately one-third of the participants refused implants. It appears that the fairly high proportions of individuals who say that they would never consider receiving dental implants, as reported in different cross-sectional studies (eg, Kronstrom et al., 2002), ${ }^{6}$ cannot be disregarded simply because of inadequate patient education. It also underscores the value of appraising cross-sectional studies for addressing questions about patient values and preferences. 
Future clinical trials in prosthodontics should focus on patientcentred outcomes and use study designs that take into account patient and clinician treatment preferences. ${ }^{7}$ Moreover, when calculating study power, it is necessary to allow for the not-insignificant proportion of patients who will refuse to be allocated to one or other arms of a trial.

\section{Asbjørn Jokstad \\ Department of Prosthodontics, University of Toronto Faculty of Dentistry, Toronto, Canada}

1. Zelen M. A new design for randomized clinical trials. N Engl I Med 1979; 300:1242-1245.

2. Adamson J, Cockayne S, Puffer S, Torgerson DJ. Review of randomised trials using the post-randomised consent (Zelen's) design. Contemp Clin Trials 2006; 27:305-319.
3. Verheggen FW, Nieman FH, Reerink E, Kok GJ. Patient satisfaction with clinical trial participation. Int J Qual Health Care 1998; 10:319-330.

4. Feine JS, Awad MA, Lund JP. Rejoinder to Bradley: Patient preferences and clinical trial design and appreciation and critique of a paper by Feine, Awad and Lund. Community Dent Oral Epidemiol 1999; 27:89-92.

5. Walton IN, MacEntee MI. Choosing or refusing oral implants: a prospective study of edentulous volunteers for a clinical trial. Int J Prosthodont 2005; 18:483-488

6. Kronstrom M, Palmqvist S, Soderfeldt B, Vigild M. Utilization of dental health services among middle-aged people in Sweden and Denmark. Acta Odontol Scand $2002 ; 60: 276-280$

7. King M, Nazareth I, Lampe F, et al. Conceptual framework and systematic review of the effects of participants' and professionals' preferences in randomised controlled trials. Health Technol Assess 2005; 9 (35).

Evidence-Based Dentistry (2006) 7, 81-82. doi:10.1038/sj.ebd.6400446

\section{EDITOR'S NOTE}

\section{Mineral trioxide aggregate in primary molar pulpotomies}

In their commentary on the paper by Holan et al., ${ }^{1}$ Duggal and $\mathrm{Al}$ Ansary ${ }^{2}$ indicated that, "In addition to including teeth with pulp exposure, asymptomatic teeth with deep carious lesions were also included in the study". This interpretation of the study is in keeping with the listing of symptomless primary molars with deep carious lesions as one of the inclusion criteria in the original paper. The inclusion criteria as listed in the article were: 1) symptomless primary molars with a deep carious lesion; 2) exposure of a vital pulp by caries; 3) no clinical or radiographic evidence of pulp degeneration, such as excessive bleeding from the root canal, internal root resorption, inter-radicular and/or periapical bone destruction, swelling or sinus tract; and 4) the possibility of proper restoration of the teeth. The authors clarified that only teeth that met all four criteria were included in the study. Thus, teeth with deep carious lesions were but without pulp exposure were not included in the study.

1. Holan G, Eidelman E, Fuks AB. Long-term evaluation of pulpotomy in primary molars using mineral trioxide aggregate or formocresol. Pediatr Dent 2005; 27:129-136. 2. Duggal M, Al Ansary M. Mineral trioxide aggregate in primary molar pulpotomies. Evid Based Dent 7:35-36. 